\title{
Was ist nötig, was ist möglich? Neuordnung der Verantwortungen in der Heilkunde aus Sicht des Deutschen Pflegerates
}

\author{
Franz Wagner
}

(C) Springer-Verlag Wien 2012

Die Berufe nach Alten- bzw. Krankenpflegegesetz sind Heilberufe gemäß Artikel 74 (19) Grundgesetz. In beiden Berufsgesetzen sind eigenverantwortliche Aufgaben als Ausbildungsziele formuliert. In der Gesundheitsversorgung ist im Rahmen arbeitsteiliger Prozesse eine Delegation ärztlicher Aufgaben verbreitet. Häufig verwischen sich die Grenzen zwischen (formaler) Delegation und (informeller) Substitution. Die Praxis ist hier schon weiter als die Rechtslage und die Verbände.

Die Versorgungsrealität erfordert eine Veränderung der Kooperation und der Aufgabenverteilung der Gesundheitsprofessionen. Das haben der Sachverständigenrat zur Begutachtung der Entwicklung im Gesundheitswesen (u. a. im Gutachten 2007) und auch der Bundestag (u. a. 2008 im Pflegeweiterentwicklungsgesetz) festgestellt. Dies betrifft nicht nur Ärzte und Pflegefachpersonen! Leider fehlt bisher ein Gesamtkonzept für eine sinnvolle Aufgaben- und Verantwortungsverteilung. Die Diskussion wird vor allem von der Sorge um Verteilung der Budgets befeuert.

Mit der Möglichkeit für Modelle der Heilkundeübertragung nach $\S 63$ (3c) SGB V hat der Gesetzgeber einen Weg, eine neue Aufgabenverteilung zu erproben, eröffnet. Allerdings ist dieser Weg extrem komplex. Keine der Gegenstrategien zur Verhinderung der Heilkundeübertragung hat aber bisher zufriedenstellende Resultate gebracht. Es stellt sich die Frage, ob die Versorgungsbedarfe uns Zeit lassen werden, Übertragung zu erproben oder ob wir nicht in absehbarer Zeit aus schierer Not solche Lösungen einführen müssen.

Grundsätzlich gilt, dass Veränderungen immer vom Klienten her gedacht und entwickelt werden müssen. Denn für deren gute Versorgung stehen Ärzte wie Pflegefachpersonen in der Verantwortung.

F. Wagner $(\bowtie)$

Bundesgeschäftsführer DBfK e. V.,

Vize-Präsident DPR e. V., Berlin 